\title{
Strategies to reduce long-term postchemoradiation dysphagia in patients with head and neck cancer: An evidence-based review
}

Vinidh Paleri, MS, FRCS (ORL-HNS), ${ }_{1 *}$ Justin W. G. Roe, MSc, ${ }^{2}$ Primož Strojan, MD, ${ }^{3}$ June Corry, MD,${ }^{4}$ Vincent Grégoire, MD, PhD, ${ }^{5}$ Marc Hamoir, MD, ${ }^{6}$ Avraham Eisbruch, MD, ${ }^{7}$ William M. Mendenhall, MD, ${ }^{8}$ Carl E. Silver, MD, ${ }^{9}$ Alessandra Rinaldo, MD, FRCSEd ad hominem, FRCS (Eng, Ir) ad eundem, FRCSGlasg,,$^{10}$ Robert P. Takes, MD, PhD, ${ }^{11}$ Alfio Ferlito, MD, DL0, DPath, FRCSEd ad hominem, FRCS (Eng, Glasg, Ir) ad eundem, FDSRCS ad eundem, FHKCORL, FRCPath, FASCP, IFCAP10

${ }^{1}$ Department of Otolaryngology-Head and Neck Surgery, Newcastle upon Tyne Hospitals, Newcastle upon Tyne, United Kingdom, ${ }^{2}$ Head and Neck Unit, The Royal Marsden Hospital NHS Foundation Trust, Fulham Road, London, UK, ${ }^{3}$ Department of Radiation Oncology, Institute of Oncology, Ljubljana, Slovenia, ${ }^{4}$ Division of Radiation Oncology, Peter MacCallum Cancer Centre, Melbourne, Victoria, Australia, ${ }^{5}$ Department of Radiation Oncology, Université Catholique de Louvain, St Luc University Hospital and Cancer Center, Brussels, Belgium, ${ }^{6}$ Department of Head and Neck Surgery, Université Catholique de Louvain, St Luc University Hospital and Cancer Center, Brussels, Belgium, ${ }^{7}$ Department of Radiation Oncology, University of Michigan, Ann Arbor, Michigan, ${ }^{8}$ Department of Radiation Oncology, University of Florida, Gainesville, Florida, ${ }^{9}$ Departments of Surgery and Otolaryngology-Head and Neck Surgery, Albert Einstein College of Medicine, Montefiore Medical Center, Bronx, New York, ${ }^{10}$ ENT Clinic, University of Udine, Udine, Italy,

${ }^{11}$ Department of Otolaryngology-Head and Neck Surgery, Radboud University Nijmegen Medical Centre, Nijmegen, The Netherlands.

Accepted 8 January 2013

Published online 16 May 2013 in Wiley Online Library (wileyonlinelibrary.com). DOl 10.1002/hed.23251

ABSTRACT: Background. Swallowing dysfunction following chemoradiation for head and neck cancer is a major cause of morbidity and reduced quality of life. This review discusses 3 strategies that may improve posttreatment swallowing function.

Methods. The literature was assessed by a multiauthor team that produced evidence-based recommendations.

Results. (1) Prospective and randomized studies with small cohorts show a trend toward benefits for a preventative exercise program addressing oral and pharyngeal structures. (2) Prospective and retrospective data indicate that better swallowing outcomes are likely when nasogastric tubes are used in preference to gastrostomy tubes to supplement enteral nutrition during chemoradiation. (3) Emerging prospective data with mature results on small cohorts support the hypothesis that radiation dose restriction to swallowing structures using intensity-modulated radiation therapy techniques leads to better swallow outcomes.

Conclusions. This study discusses 3 strategies for improving swallowrelated outcomes in patients undergoing chemoradiation for head and neck cancer and identifies areas for future research. (C) 2013 Wiley Periodicals, Inc. Head Neck 36: 431-443, 2014

KEY WORDS: dysphagia, chemoradiation, tube feeding, IMRT, swallowing exercise

\section{INTRODUCTION}

Among the most profound effects of chemoradiation (CRT) for head and neck cancer is the impact on the ability to eat and drink. It is recognized that organ-preserving CRT regimes do not necessarily result in functional swallowing preservation. ${ }^{1}$ Prospective studies have identified swallowing function as a top priority for patients with head and neck cancer both at diagnosis and after treatment. ${ }^{2}$ Dysphagia can result in dehydration and malnutrition and place people at risk of aspiration. Dysphagia is one of the key factors that can reduce quality of life (QOL), with increased severity of dysphagia correlating with increased adverse effects on QOL anxiety and depression. ${ }^{3}$ The use of CRT in the management of advanced head and neck cancers, combined with a reduc-

*Corresponding author: V. Paleri, Otolaryngology-Head and Neck Surgery, The Newcastle upon Tyne Hospitals NHS Foundation Trust, Newcastle upon Tyne, UK. E-mail: vinidh.paleri@ncl.ac.uk

This paper was written by members and invitees of the International Head and Neck Scientific Group. tion of mean age at diagnosis for some of these tumors make QOL and survivorship important considerations. This review will identify if strategies exist in the current literature to reduce dysphagia-related morbidity and assess the strengths of the available evidence and make recommendations for practice.

During treatment, almost all patients receiving CRT need nutritional supplementation because the toxicity of treatment is severe enough to impair adequate nutritional intake. ${ }^{4}$ The treatment fields often are wide to encompass a large area, including the salivary glands and swallowing musculature. Some may avoid feeding tube placement with aggressive swallowing therapy initiated at the beginning of treatment, optimal analgesia, augmented nutrition and intravenous fluids, supported by a multidisciplinary team that includes speech and language therapists and dietitians, also known as speech-language pathologists and nutritionists in the United States.

This review will comprehensively evaluate the evidence base pertaining to 3 strategies that have been shown on exploratory studies to reduce long-term swallowing morbidity. These include the use of prophylactic swallowing exercises, avoiding gastrostomy tubes (G-tubes) 
during CRT and using intensity-modulated radiation therapy (IMRT) techniques to reduce the dose delivered to swallowing-related organs at risk.

The literature on evaluation of swallowing function is hampered by the use of a variety of measures that are not comparable. In addition, several authors present only QOL data and interchangeably discuss these as functional outcomes. Although QOL measurements are affected by function, it must be noted that they do not necessarily measure function, and care should be taken when reading into the results. This report will summarize the published data as presented with appropriate commentary, but assumes that the reader is familiar with the various measures discussed.

\section{Prophylactic swallowing exercises}

Swallowing difficulties can occur as a result of acute and late treatment effects. ${ }^{5}$ Acutely, patients may experience mucositis, edema, and erythema, compounded by taste changes, nausea, and xerostomia. This has an impact on the ability and desire to swallow, and disuse of the skeletal muscles involved in swallowing during treatment may cause weakness. The importance of continuing with even limited oral intake during treatment has been highlighted in the literature. ${ }^{6}$

Radiation-induced fibrosis in the swallowing structures can occur due to abnormal tissue healing following treatment with hyperactivation of transforming growth factor beta (TGF- $\beta$ ) and connective tissue growth factor (CTGF). Fibrotic connective tissue wraps around muscle fibers, resulting in restricted movement and, subsequently, muscles fibers become weak due to disuse atrophy. Although some patients are able to resume eating and drinking after acute treatment effects resolve, others continue to experience difficulties. Swallowing difficulties can result from the gradual onset of fibrosis or sudden, late-onset fibrosis in those who have historically managed well following treatment.

The concept of exercises to reduce long-term swallowing-related morbidity has been explored in several recent studies. Early implementation of protective exercises has been highlighted as an important intervention for patients undergoing organ-preservation protocols for a number of years, given the known impact of CRT on swallowing function. However, it is only recently that studies have started exploring the potential benefit of prophylactic swallowing exercises for patients being treated with radiotherapy. Despite the limited evidence base for prophylactic swallowing exercises, the implementation of the exercise programs has become an important component of the management of patients undergoing organ-preservation treatments. In the United Kingdom, 50\% $(n=21 /$ 42) of speech and language therapist teams see patients routinely prior to treatment with $71.4 \%(n=30 / 42)$ of speech and language therapist teams recommending prophylactic swallowing exercises. ${ }^{7}$ This compares with $18.3 \%$ of 759 individual speech and language therapists in the United States. ${ }^{8}$ In the United States it was reported by $70.6 \%$ of speech and language therapists that there is no institutional policy for referrals or referrals are made on a case-by-case basis. ${ }^{8}$ This difference may be accounted for given that UK-based speech and language therapists working in the National Health Service are core members of the head and neck multidisciplinary team as stated in national improving outcomes guidance. ${ }^{9}$ Therefore, they are invariably involved in patient care at the outset.

To date there have been 6 studies published evaluating prophylactic swallowing exercises and swallowing outcomes including 3 randomized controlled trials (RCTs). Despite the increased number of studies, a variety of measures have been used to understand the potential impact of prophylactic swallowing exercises on swallowing (see Table 1). In each of the published studies, the exercise prescriptions differ with exercise regimes initiated at varying points, which are either unspecified, 2 weeks prior, or on the first day of treatment. Exercise schedules also vary between studies and relied either on a self-initiated exercise schedule or a program administered under the direct supervision of a speech and language therapist. Details of these exercise regimes are presented in Table 1. The variety of reported regimes and exercise prescriptions may explain the inconsistency in swallowing therapy practice patterns reported in the United States and the United Kingdom. ${ }^{7,8}$ In addition, a variety of outcome measures have been reported with only 2 providing baseline and posttreatment instrumental measures. ${ }^{10,11}$ Another important consideration is the difference in prophylactic versus reactive tube feeding regimes between centers (see Table 1) and how that might have an impact on swallowing.

The first studies in this area were initiated at the same center. $^{12,13}$ Kulbersh et al ${ }^{12}$ studied 37 patients who underwent primary radiation or combined CRT treatment for newly diagnosed hypopharyngeal, laryngeal, or oropharyngeal primary tumors. In this prospective cohort analysis and cross-sectional QOL analysis, swallowing exercises were taught to 25 patients 2 weeks prior to the start of radiation. The MD Anderson Dysphagia Inventory $(\text { MDADI })^{14}$ was administered an average of 14 months after treatment to assess the success of the protocol. Patients who performed pretreatment swallowing exercises $(n=25)$ showed improvement in the overall MDADI score $(p=.0002)$ compared with the control population ( $n=12$ ) who underwent posttreatment therapy. Furthermore, a separate analysis of individual domains of the MDADI (global, emotional, functional, and physical) demonstrated improved QOL.

In a retrospective case control study, Carroll et al $^{13}$ compared patients who completed a prophylactic exercise program $(n=9)$ with those completing a posttreatment regime $(n=9)$. A videofluoroscopy (VFS) was carried out 3 months after treatment. Significant differences in epiglottic inversion $(p=.02)$ and tongue base position during the swallow $(p=.025)$ were observed in the prophylactic exercise group over the control group. The limitation of this study is the lack of baseline swallowing measures given the potential impact of cancer on pretreatment swallowing function. ${ }^{15-17}$

A more recent, prospective nonrandomized study ${ }^{18}$ has challenged the benefit of preventative swallowing rehabilitation. Ahlberg et $\mathrm{al}^{8}$ compared 190 patients receiving preventive rehabilitation with 184 patients in the control group on a range of measures detailed in Table 1. Despite the admirable sample size, this heterogeneous group 


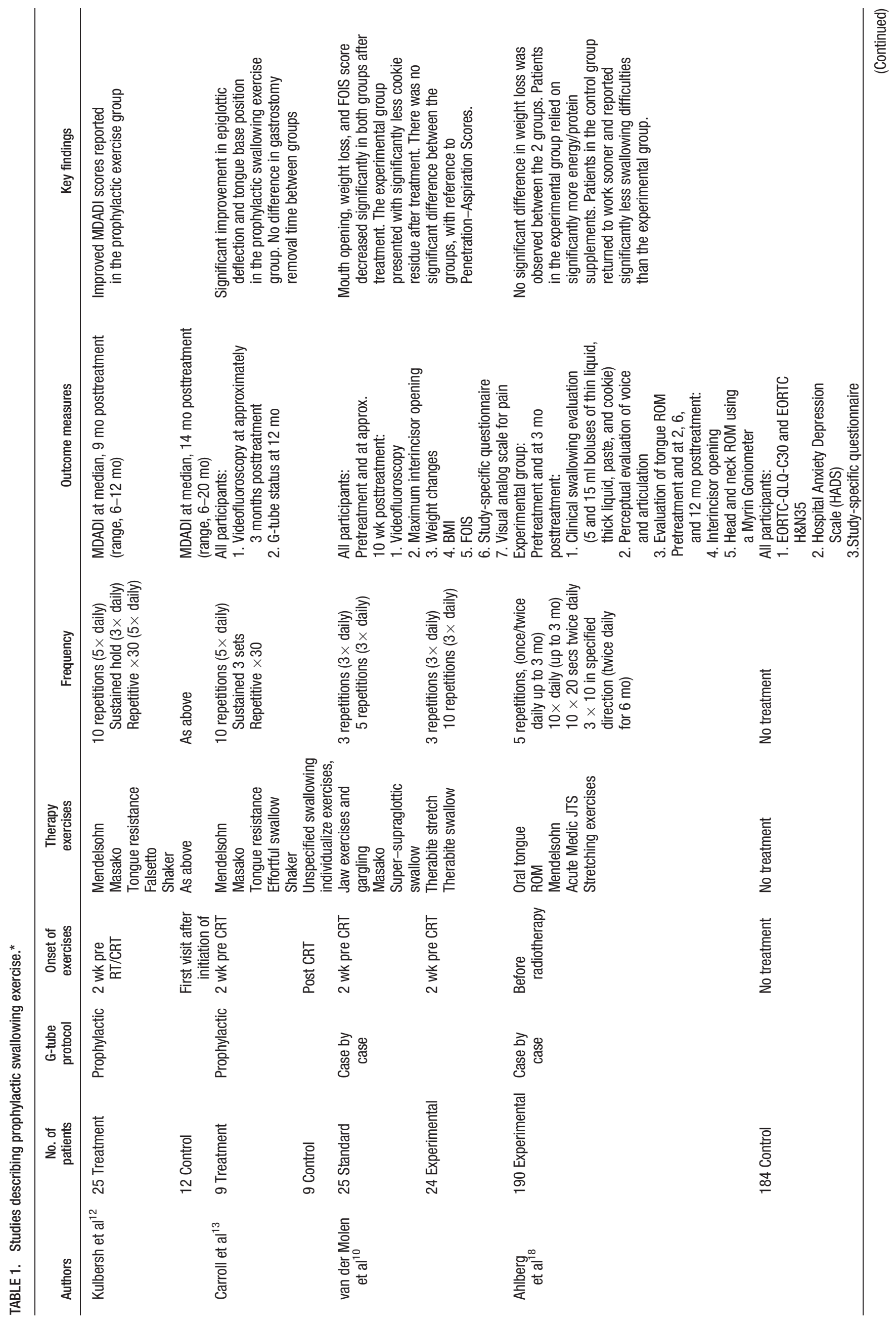




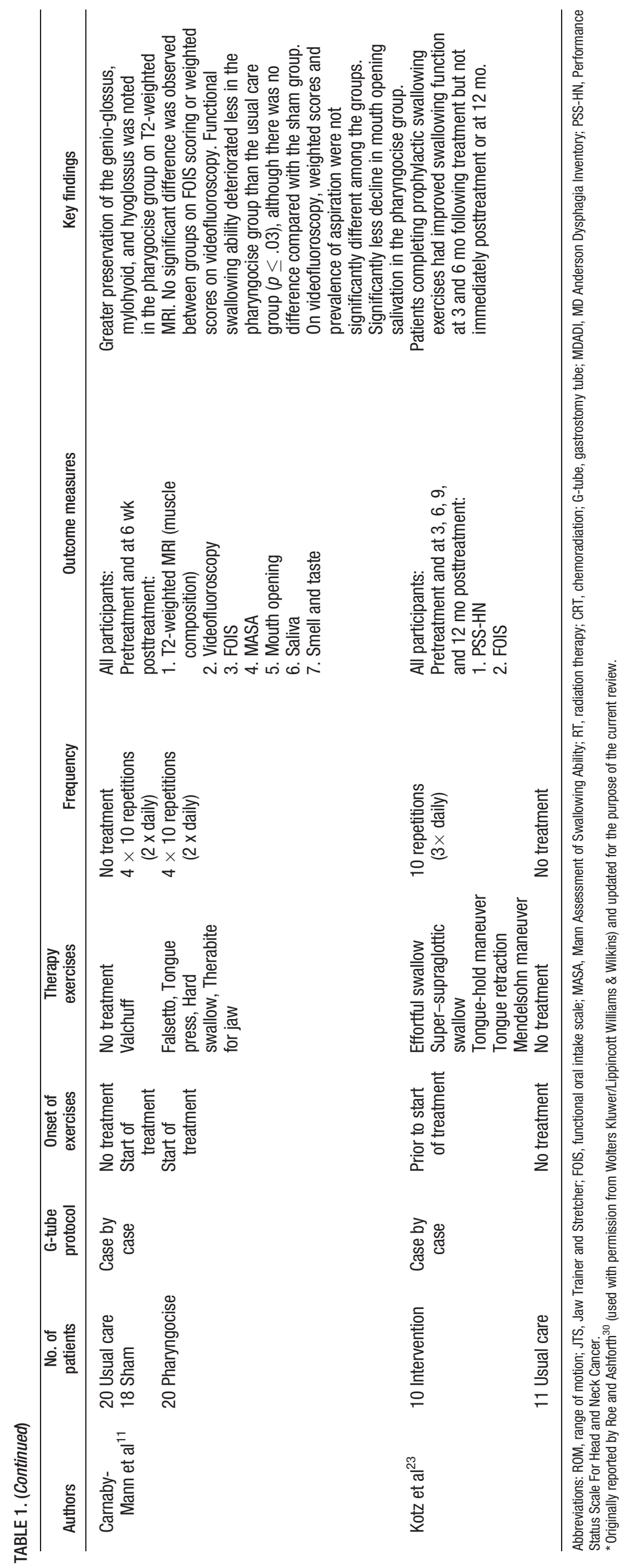


included patients who had undergone major surgery prior to receiving radiotherapy with or without chemotherapy. Brachytherapy was also used in certain cases. Although a range of measures were comparable across groups, only the experimental group underwent more detailed evaluation, including a clinical swallowing evaluation. Speech and language therapy measures were collected at baseline and 3 months. Physiotherapy measures were collected at 2, 6, and 12 months after treatment completion. The project-specific questionnaire focused on self-reported functional losses and also contained questions about rehabilitation and working ability. Interestingly, patients in the control group reported significantly less swallowing difficulties than those in the study group, with a proportional odds ratio (OR) of 2.3 (95\% confidence interval [CI]: 1.3-4.0). In the control group, 58\% answered that they could swallow all consistencies of food compared with $35 \%$ in the study group $(p<.001$; data not shown in tables). There was a significantly higher number of patients using high energy/protein supplements in the study group (60\%) than in the control group $(32 \%)(p<$ .001). The authors discuss the possibility that patients in the study group may have been more aware of swallowing problems and nutritional issues and may have had higher expectations regarding their functional status. The total incidence of reported swallowing problems was $61 \%$ $(114 / 185)$. No parameters in the European Organisation for Research and Treatment of Cancer (EORTC) questionnaire $^{19}$ or the Hospital Anxiety Depression Scale $(\mathrm{HADS})^{20}$ showed any significant difference between the 2 groups at 6 months after termination of treatment.

van der Molen et $\mathrm{al}^{10}$ published the first RCT on 55 patients with various head and neck primaries undergoing CRT to a standard or an experimental preventive rehabilitation arm (see Table 1). They specify that these patients received IMRT. Complete posttreatment data were available for 49 patients. Structured multidimensional assessment was performed before and 10 weeks after CRT (see Table 1). This study did not include a "no intervention group," given that Dutch national guidelines deem it unethical to withhold rehabilitation. Only $4 \%(n=2)$ of patients were tube fed prior to treatment and, although this increased to $76 \%(n=38)$ by the end of radiotherapy, by the 10 -week point this had reduced to $37 \%(n=$ 18). Compared with similar CRT studies at the same institution, fewer patients were still tube-dependent after CRT. They concluded that exercises were helpful in reducing the extent and/or severity of various functional short-term effects of CRT. This group recently presented their 2-year posttreatment results and found that no significant functional differences were found between the 2 preventative exercise groups. ${ }^{21}$ However, functional difficulties were limited when compared with a previous trial where exercises were not implemented.

Carnaby-Mann et $\mathrm{al}^{11}$ conducted an RCT comparing the effectiveness of preventative behavioral intervention for dysphagia. Patients were treated with conventional radiotherapy or IMRT with or without chemotherapy. Patients were randomized to 3 arms: usual care (UC), sham exercises, and high intensity swallowing therapy ("pharyngocise"). The UC group were evaluated by the radiation oncologist and received supervision for feeding and safe swallowing precautions and received weekly phone reviews from a research assistant. The sham group carried out a buccal extension exercise and dietary modification as required. The latter was comprised of a battery of exercises for the swallowing musculature (see Table 1). These included exercises such as tongue press, hard swallow, and jaw resistance/strengthening using the Therabite Jaw Motion Rehabilitation System. Both the sham exercise and swallow exercise group performed repetitions of their exercise schedule for 45 minutes, twice daily for the duration of the treatment under the supervision of the study speech and language therapists.

A potential reduction in loss of muscle structure due to prophylactic swallowing exercises has been noted incidentally in the literature. ${ }^{13,22}$ This was addressed in the study reported by Carnaby-Mann et al., ${ }^{11}$ the primary outcome measure being the change in size of the muscles of the tongue and floor of mouth (mylohyoid, hyoglossus, and genioglossus) and the pharyngeal constrictor muscles on T2-weighted magnetic resonance imaging (MRI) scans at 3 time points: before treatment, after treatment, and at 6 months after randomization. The results of this study showed less structural loss in the genioglossus, hyoglossus, and mylohyoid muscles in the group receiving high intensity swallowing therapy; the study did not report the results for the constrictor muscles due to movement artifact. Patient outcome measures showed the functional swallowing ability deteriorated less in the pharyngocise group than in the UC, with this being statistically significant $(p \leq .03)$; there was no difference compared with the sham exercises group $(p<.06)$. They also reported that MRI results for the sham group fell between the control and pharyngocise group. Given these data, the authors suggest that there may be benefit to lower intensity exercise programs. The Functional Oral Intake Scale (FOIS) scores did not change significantly among the groups. During treatment, it was found that patients in the pharyngocise group maintained oral feeding more often than those in the UC group (42\% vs 14\%) and fewer required gastrostomy feeding than the no intervention group $(20 \%$ vs $30 \%)$. The authors report that their data were most complete at 6 weeks posttreatment as withdrawal rates at 6 months prevented meaningful analysis of the data.

An RCT was reported recently outlining a prophylactic exercise program versus a UC approach in 26 patients undergoing CRT. ${ }^{23}$ Swallowing function was evaluated using the FOIS ${ }^{24}$ and Performance Status Scale for Head and Neck Cancer (PSS-HN) ${ }^{25}$ at 3, 6, 9, and 12 months. The intervention group completed exercises during treatment (see Table 1) and a weekly therapy session was provided to improve program adherence and check technique. They found that 9 of the 13 patients in the intervention group were able to complete their exercises throughout treatment, although 4 of them ceased exercises in week 4 and another 5 by week 5 of their treatment. Patients ceased treatment due to pain and fatigue. Significant differences were noted between groups with the intervention group having improved FOIS and PSS-HN Normalcy of Diet scores at 3 and 6 months but not in the immediate posttreatment period or at 9 and 12 months. Twelve patients required gastrostomy feeding, although no statistical difference in placement between groups was 
reported and the median removal time was 3 months. The authors recognize the limited sample size in this study and the need for a larger number of patients for future studies.

In these times of financial austerity, it is crucial that work has been reported on the potential cost effectiveness of rehabilitation programs in Europe and the United States. $^{26,27}$ Using the data from their RCT, ${ }^{10}$ researchers at the Netherlands Cancer Institute have developed a Markov decision model of a preventative exercise program (PREP) versus UC. ${ }^{27}$ The UC data were acquired from previous RCTs at their center comparing intra-arterial and intravenous CRT. ${ }^{28,29}$ Although it was found that PREP was more cost effective than UC, the authors highlight that results are based on preliminary data and limitations exist such as comparable disease subsites between the groups and radiotherapy technique. Recently presented data from the United States also used a Markov model to estimate the incremental cost-effectiveness ratio of a prophylactic exercise program versus a posttreatment program in 100 patients with hypothetical head and neck cancer. $^{26}$ They found that a preventative program of swallowing exercises in patients receiving CRT was clinically beneficial and led to significant cost savings over usual, posttreatment care. It should be noted that accurate assessment of cost effectiveness relies on good evidence for the incremental benefit of a treatment because it is not possible to accurately populate the transition probabilities in a Markov model. However, these studies have paved the way for others to evaluate the cost-effectiveness of their rehabilitation interventions as more data become available.

In each of these studies, the researchers have made a significant contribution to our understanding of how prophylactic swallowing exercises may influence the swallowing outcome. This area of study is developing and, given the limited evidence base, clinicians have adopted what they feel to be the most appropriate intervention based on published data and clinical experience. ${ }^{30}$ At this stage, the results of these studies present the clinician attempting to translate research findings into clinical practice with a number of questions. When should exercises start? Which exercises should patients do? How frequently should they carry them out? How long should patients continue exercises for? What are the long-term benefits of prophylactic swallowing exercises?

Adherence to exercises and the support needed by patients is a key issue to understand the true benefit and feasibility of these programs. Three of the published studies reported how patient adherence was ascertained. ${ }^{10,11}$ The significant toxicity experienced during treatment may have an impact on the individual's ability to carry out exercises. Pretreatment counseling by speech and language therapists is crucial and patients should understand the rationale and potential benefits of swallowing rehabilitation so that they become motivated rehabilitation partners. $^{30}$ Recently presented data from an RCT in the United States have shown that improved outcomes can be achieved in a therapist-assisted exercise program over self-directed exercises. ${ }^{31}$ Furthermore, the presence of depression and fatigue prior to starting prophylactic exercises can influence adherence to programs. ${ }^{32}$ This further supports the need for detailed, multidimensional evaluation of patients' pretreatment to improve swallowing outcomes.

In the absence of multidimensional pretreatment evaluation (including instrumental assessment), it is not possible to specify which exercises may be the most beneficial for individuals. Further longitudinal studies with greater numbers are required to understand the relative benefits of strengthening and range of motion exercises on swallowing outcome. In addition, there should be clarity on the most effective exercise programs, including dose-response data. Speech and language therapists recognize the importance of tailoring swallowing rehabilitation to the individual (often impossible to do in a clinical trial) both prior to and following treatment based on information acquired through detailed swallowing evaluation. ${ }^{7}$ In addition to exercise, it is important that the effect of other treatments are explored such as lymphedema therapy, massage, and stretching. ${ }^{33}$ Limitation of significant variables such as excluding patients who have undergone significant surgical resection is essential given the swallowing difficulties that may result. To understand the potential effects of prophylactic exercise regimes on swallowing outcome, researchers should clearly document the tube feeding approach at their center and whether patients received conventional or IMRT. The implications of these factors are discussed in the following sections.

\section{Use of gastrostomy tubes}

Prophylactic gastrostomy placement is associated with several benefits to patients undergoing CRT. These include a wider portal for nutritional supplementation that is less prone to blockage and, from a patient perspective, can be covered by clothing. On the contrary, nasogastric (NG) tubes are beset by problems of easy blockage and cannot be concealed. The indications, benefits, and drawbacks of a pretreatment gastrostomy have been reviewed in detail in the literature. ${ }^{34}$ It is reasonable to say that no consensus exists on several key issues. However, this review will focus on the issue of adverse effects, if any, that gastrostomy use may have on the swallowing performance after treatment.

Gastrostomy placement does reduce the need for the patient undergoing CRT to swallow to maintain nutrition. Thus, it is likely that patients using G-tubes exhibit a reduction in use of the swallowing musculature. This reduction in use of the swallowing musculature, combined with the mucositis caused by radiation, has been hypothesized to increase the risk of fibrosis in the muscles and pharyngoesophageal stricture. Data from prospective studies suggest that patients tend to retain Gtubes longer than NG tubes. ${ }^{35-37}$ On this basis, some centers have moved away from prophylactic G-tubes, with the assumption that patients avoid oral intake earlier, if an alternative feeding route is available.

Four retrospective studies, ${ }^{38-40}$ one prospective study, ${ }^{36}$ and one randomized trial ${ }^{35}$ have explored this hypothesis. Mekhail et $\mathrm{al}^{38}$ retrospectively compared $29 \mathrm{NG}-\mathrm{fed}$ patients with 62 G-tube fed patients, finding that G-tube fed patients had more dysphagia at 3 months (59\% vs $30 \% ; p=.015)$ and at 6 months $(30 \%$ vs $8 \% ; p=.029)$. The median tube retention duration was also higher for 
the G-tube group. However, this study used a gross dysphagia grading system, with limited long-term follow-up, so the impact on swallowing impairment and tube selection remains unknown. There may be other "unknown" patient factors that are difficult to capture such as levels of determination and prioritization attributed to eating, affecting tube duration. In this study, 19 G-tube-fed patients have been shown to require pharyngoesophageal dilatation more frequently compared with NG-tube-fed patients (23\% vs $4 \%$; $p=.022)$.

Oozeer et $\mathrm{al}^{39}$ retrospectively compared patient-reported swallowing outcomes, using the MDADI, between 2 groups treated by primary CRT: 1 group received nutritional supplementation via a prophylactic G-tube and the other by the oral route or as required via a NG tube. The groups were matched for age, site, and stage of tumor and were disease-free at least 24 months following treatment. The prophylactic gastrostomy group consisted of 16 patients and the oral/NG tube group of 15 patients. There were statistically significant differences in MDADI scores between the 2 groups in all domains of the questionnaire $(p<.001)$, with superior outcomes in the NG group. They concluded that use of G-tubes during CRT conferred a worse swallowing outcome in the long term in this tightly matched cohort of patients.

Chen et $\mathrm{al}^{40}$ retrospectively studied 120 consecutive patients treated with CRT for Stage III/IV head and neck cancer, with the most common primary site in the cohort being the oropharynx (66 patients). Sixty-seven patients $(56 \%)$ were treated using IMRT. Seventy patients $(58 \%)$ received prophylactic gastrostomy placement. They found that prophylactic gastrostomy placement reduced mean weight loss during radiation therapy from 43 pounds (range, 0-76 pounds) to 19 pounds (range, 0-51 pounds), and this reduction was statistically significant $(p<.001)$. However, the proportion of patients who were gastrostomy dependent at 6 and 12 months after treatment was higher for those who received prophylactic G-tube (41\% and $21 \%$, respectively), compared with those who did not receive prophylactic G-tube ( $8 \%$ and $0 \%$, respectively); this difference was statistically significant $(p<.001)$. Additionally, prophylactic G-tube was associated with a significantly higher incidence of late esophageal stricture compared with those who did not have prophylactic Gtube $(30 \%$ vs $6 \%, p<.001)$.

The above studies, although retrospective, show clear trends for better swallowing in those who do not receive prophylactic gastrostomy. It is feasible that the presence of a NG tube or continued oral swallowing uses the muscles of swallowing more frequently, leading to reduction in muscle fibrosis and thus a better swallowing outcome.

There are 2 prospective studies on the subject of tube feeding and swallowing outcomes. In a prospective study comparing swallowing outcomes using the Common Toxicity Criteria, version 2.0 (CTC v2.0) dysphagia grade in 32 G-tube fed patients and 73 NG-fed patients, Corry et $\mathrm{al}^{36}$ found that there was more grade 3 dysphagia in Gtube fed disease-free survivors at 6 months $(25 \%$ vs $8 \%$, $p=.07)$. These authors preceded this prospective cohort with a RCT that was terminated due to poor recruitment. In the randomized trial, ${ }^{35} 18$ received an NG tube and 15 a G-tube. This study found no statistically significant difference in the rates of CTC v2.0 grade 3 dysphagia at 6 months post-RT (ie, ongoing use of feeding tube). There were 4 of 15 patients in the G-tube group with grade 3 dysphagia compared with only 1 of 18 in the NG-tube group $(p=.15)$.

One potential benefit of $\mathrm{NG}$ tubes is a reduction in post-cricoid stricture formation, due to the tube acting as a stent. Complete obliteration of the lumen has been reported with G-tube use, but never with NG tubes. ${ }^{38,40,41}$

There is no doubt that patients with head and neck cancer may present with swallowing difficulties due to their cancer at diagnosis. ${ }^{16,17}$ They may be at risk of aspiration and its sequelae and nutritional compromise and may require placement of a feeding tube prior to treatment. Other patients may not have any dysphagia and specialist multidisciplinary support during treatment including optimal symptom control, swallowing rehabilitation, and nutritional advice are crucial. Appropriate selection of patients who may require tube feeding is crucial and should be based on best evidence, detailed speech and language therapist and dietetic evaluation, and patient preference in a multidisciplinary setting. Avoiding or reducing prophylactic gastrostomy use may not be possible in several clinical scenarios. Examples include patients with significant pretreatment dysphagia, those anticipated to require $>8$ weeks enteral nutrition, and/or with very advanced (usually T4) primary tumors, especially in the hypopharynx. A recent study has found that even if tube feeding is implemented, patients who continued with even limited oral intake maintained significantly improved swallowing function over those who relied exclusively on tube feeding. ${ }^{6}$ Another important consideration is the fact that many head and neck cancer patients eat despite evidence of dysphagia or aspiration on instrumental evaluation. Therefore dysphagia burden may be underestimated if judged solely on the basis of the presence of gastrostomy. 42

\section{IMRT as a strategy to reduce swallowing dysfunction}

The rationale for using the IMRT technique as a strategy to avoid dysphagia is based on the established relationship between functional status of the swallowingrelated structures and the pattern of irradiation dose distribution in these structures and on the ability of the IMRT to shape the high-dose volume in accord with the 3dimensional outline of the target(s). This way, nearby structures not infiltrated with cancer cells are irradiated with lower doses, which allow at least partial preservation of their function. Recently reported results of the PARSPORT study, a RCT that found parotid-sparing IMRT a valuable method for reducing the incidence of severe xerostomia, can be considered as a proof of principle. ${ }^{43}$

The pioneering work of Eisbruch et $\mathrm{al}^{44}$ recognized that muscular components of the swallowing apparatus, critical to the development of dysphagia in irradiated patients, can be spared by IMRT. Studies have found significant correlation with dysphagia/aspiration and various dosevolume parameters for the pharyngeal constrictor muscles (superior, medial, and inferior group), esophageal inlet, and glottic and supraglottic larynx. ${ }^{45-48}$ Other authors have confirmed these findings, with steep dose-response 
relationships between dose to different parts of swallowing apparatus and dysphagia in the short to medium term (Table 2). For instance, Levendag et $\mathrm{al}^{49}$ found a $19 \%$ increase in the probability of late dysphagia grade $3 / 4$ ( $>3$ months after completion of the therapy) with every additional $10 \mathrm{~Gy}$ after a dose of $55 \mathrm{~Gy}$ in superior constrictor muscles, whereas Caudell et $\mathrm{al}^{50}$ reported a $7 \%$ to $11 \%$ increase in risk for gastrostomy dependence or aspiration with every $1-$ Gy increase in a mean dose to the larynx or inferior constrictor. Recently, van der Laan et al ${ }^{51}$ compared in their planning study 30 standard IMRT treatment plans with swallowing-sparing IMRT plans that aimed to reduce the dose to organs at risk for swallowing dysfunction in the same patients. The dose characteristics of the target volumes and normal structures, including parotid glands, were not allowed to differ between the 2 planning techniques. Adequate coverage of target volumes and dose to critical structures within acceptable limits were achieved, whereas the mean doses to the various swallowing-related structures were reduced, depending on $\mathrm{N}$ classification and primary tumor location. In addition, the observed dose reductions were reflected in reduced estimates of the normal tissue complication probability values for both, physician-rated [Radiation Therapy Oncology Group (RTOG) grade 3/4, for 9\%] and patient-rated measures for swallowing dysfunction (moderate to severe complaints: for solid food $7.9 \%$, for soft food $2.4 \%$, for liquid food $1.4 \%$, for choking when swallowing $0.9 \%$ ). However, it must be noted that this "threshold" may depend entirely on the outcome measure chosen. For instance, Eisbruch et $\mathrm{al}^{52}$ correlated doses with various outcome measures (objective and subjective outcomes) and noted varying correlation of the doses with each outcome measure. It is likely that mean pharyngeal constrictor doses above 45-60 Gy are associated with worse dysphagia.

\section{Technical considerations}

In line with the described importance of the dose-volume relationships found for structures involved in swallowing, the emerging role of IMRT planning has been underlined by several authors. Whereas a significant improvement in swallowing outcome between nonconformal 3-field technique and conformal "horseshoe" technique was described by Frowen et al, ${ }^{53}$ additional sparing of swallowing structures can be achieved with IMRT and its variants. Better sparing of the lower part of swallowing apparatus, in particular the larynx, inferior pharyngeal constrictor muscle, and esophageal inlet, was demonstrated by using split-field IMRT technique. In this technique, an anterior low-neck field with a $2 \mathrm{~cm}$ midline shielding block to protect larynx is matched with IMRT fields covering primary tumor and upper parts of the neck. Of note, compromised coverage of inferior parts of elective nodal volumes had to be acknowledged even after modification of the technique (eg, by dynamic field matching technique), which could be of particular importance in patients with extensive nodal disease and/or lowsited primaries. ${ }^{54-57}$

However, whole-field IMRT can also offer an advantage of sparing organs at risk for swallowing dysfunction. Eisbruch et $\mathrm{al}^{44}$ compared V50 (volume that received $\geq 50$ Gy of irradiation) of the previously identified dysphagia/aspiration-related structures (pharyngeal constrictor muscles, surpraglottic and glottic larynx) on the standard 3-dimensional conformal radiotherapy (3D-CRT) plan, standard IMRT plan, and dysphagia-optimized IMRT plan (with sparing of these organs included in the optimization process). All 3 plans were produced for each of 20 patients included in the study. Compared with the 3D-CRT and standard IMRT, dysphagia-optimized IMRT reduced V50 of the critical structures without compromising doses to the targets: in pharyngeal constrictors by $20 \%$ and $10 \%$ on average, respectively, and in the larynx by $18 \%$ and $7 \%$, respectively.

Furthermore, robust computed tomography (CT)-based guidelines for delineations of the most relevant swallowing organs at risk for irradiation-induced dysfunction have since been published to facilitate objective comparison and interpretation of radiotherapy sequelae between studies. $^{58}$

\section{Drawbacks of IMRT}

There are some disadvantages associated with IMRT, including a more inhomogeneous dose distribution, an increased risk of a marginal miss, increased total body dose, and increased time and expense. ${ }^{59,60}$ One of the problems associated with radiotherapy, including IMRT, is the "Jello effect," where reducing the dose to one adjacent normal tissue structure simply pushes dose toward another. Another problem is that the swallowing musculature may be close to the primary and reducing the dose to these structures might increase the risk of a marginal recurrence.

A recent review of 26 studies (10 used IMRT and 16 used conventional techniques) with 4727 patients concluded that IMRT studies reported a 3.3 fold increased risk of pharyngoesophageal stricture compared with that of conventional RT studies (OR: 3.3; 95\% CI: 2.6-4.2; $p<$ $.001)$. The study authors identify in the discussion that this could be due to higher dose received by the larynx, inferior constrictors, and cervical esophagus when whole-field IMRT is used to irradiate the low neck. Unlike conventional RT or split-field IMRT, whole-field IMRT does not use a midline block to protect the larynx, inferior constrictors, and the cervical esophagus, resulting in higher doses to these organs, unless they are specified as organs at risk and an effort is made to spare them in the optimization process. The authors accept that other confounding variables, such as taxanes, have been used in the IMRT studies and thus results should be interpreted with caution. ${ }^{61}$

Finally, because the use of IMRT and concomitant chemotherapy has increased, so has the use of percutaneous gastrostomy, which likely increases the risk of longterm swallowing problems. ${ }^{34}$

\section{Results of studies on IMRT and swallowing}

A systematic review of the literature on swallowing outcomes after IMRT by Roe et $\mathrm{al}^{7}$ (published in English between 1998 and 2009) identified 16 papers that met required inclusion criteria regarding methodologic quality and method of swallowing assessment. They concluded that a favorable swallowing outcome might be possible if 


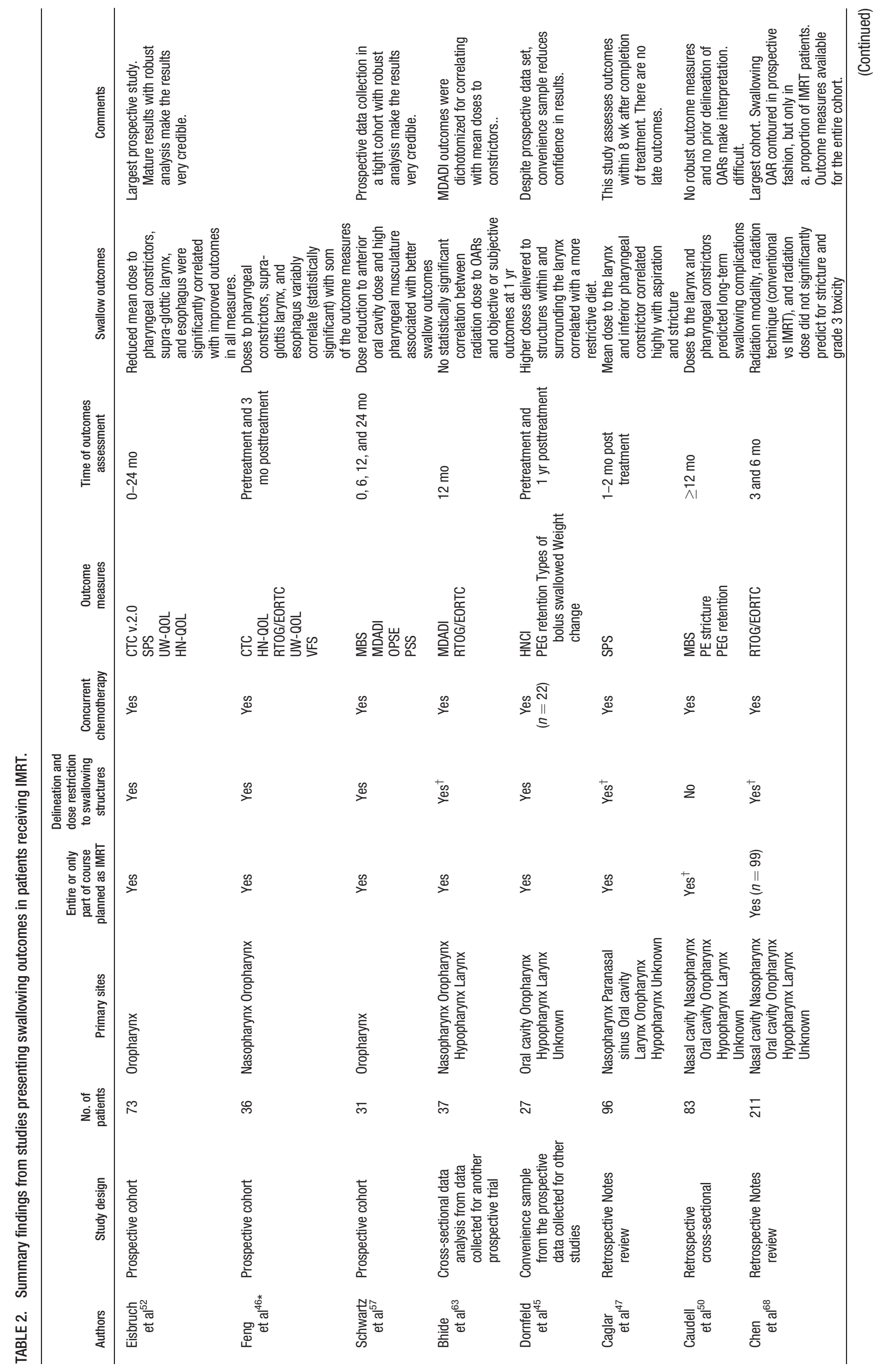




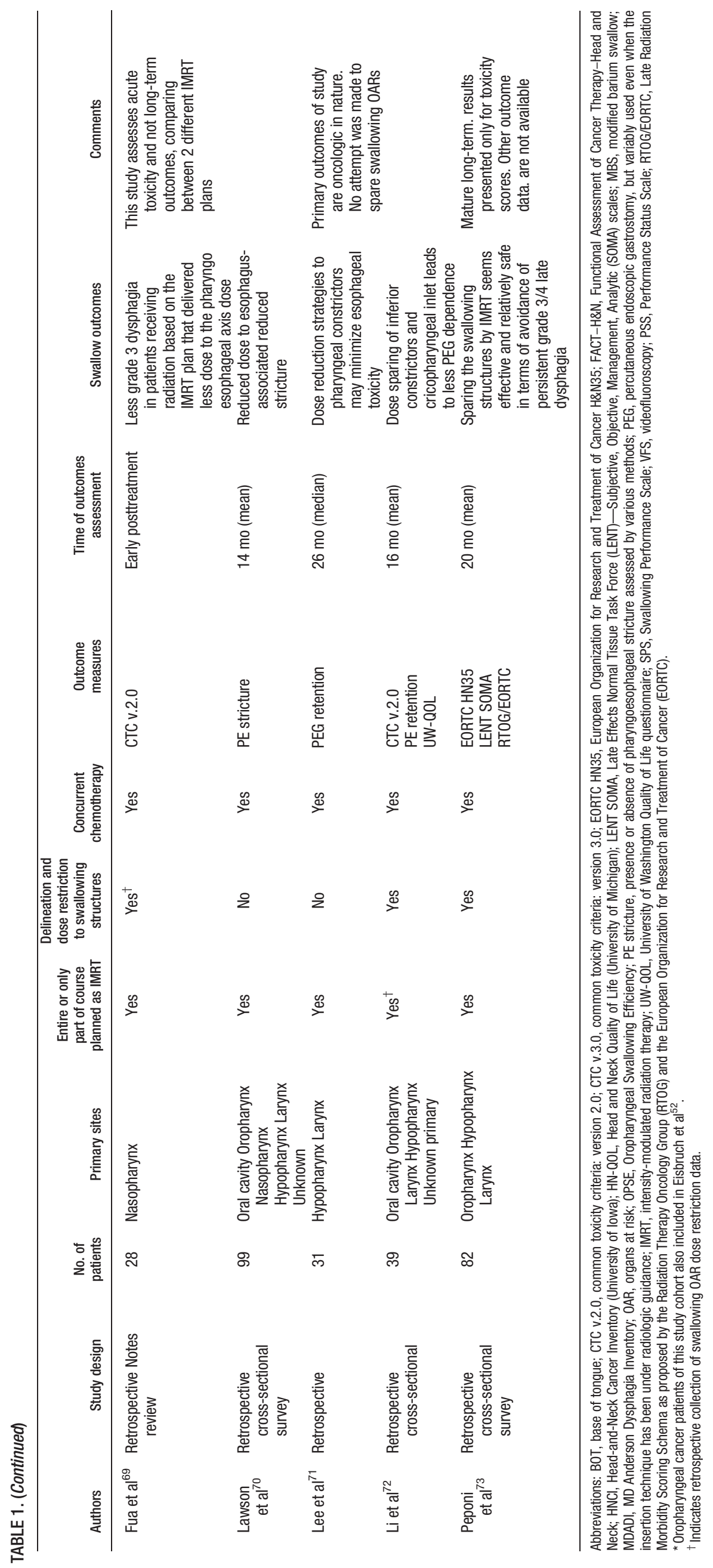


radiation dose to certain structures is limited. Table 2 summarizes the literature to date with the addition of more studies. The table includes data on the study design, the outcome measures, swallowing outcome after treatment, and pertinent comments from the current authors. It is evident that it is impossible to compare results across studies. This is due to heterogeneity in the patient population, use of a range of outcome measures that have not been shown to correlate with each other and limited use of instrumental assessment (ie, Fiber-optic Endoscopic Evaluation of Swallowing [FEES] and VFS). In addition, the methods used to delineate and reduce dose to swallowing organs at risk are not uniform.

As can be seen, this review has identified only 4 prospective studies that have used a range of outcome measures to analyze the issue and are worthy of discussion. The outcomes from the retrospective studies are summarized in Table 2.

\section{Prospective studies}

The most robust evidence for the use of dose restriction to swallowing organs at risk comes from the data published by Eisbruch et al. ${ }^{52}$ In a cohort of 73 patients with oropharyngeal cancer, followed up for up to 2 years, the study identified that increasing radiation dose the pharyngeal constrictors and supraglottic larynx correlated with worse function as measured using a variety of swallowing-related outcome measures (objective and subjective). As expected, the toxicity causing dose varied based on the outcome measure. Similar results are seen in other publications from the same center, albeit with shorter follow-up times. ${ }^{62}$

Schwartz et $\mathrm{al}^{57}$ presented similar data on a smaller cohort of 31 patients with oropharyngeal cancer enrolled in a prospective phase II trial evaluating swallowing function at baseline, 6, 12, and 24 months post-IMRT. The mean dose to the glottic larynx using an anterior field with a midline block to shield the larynx was $18 \mathrm{~Gy}$ (range, 6-39 Gy). Thirteen patients (42\%) received concomitant chemotherapy. Swallowing structures were contoured and the doses to these structures were calculated. One patient (3\%) was feeding-tube dependent at 24 months. Using the MDADI and the Oropharyngeal Swallowing Efficiency (OPSE) as outcome measures, this study performed dose-volume analyses to identify candidate dysphagia-associated organs at risk. Although the MDADI had no correlation to organ-at-risk doses, the results demonstrated that reduction of dose to the anterior oral cavity and high pharyngeal musculature resulted in better OPSE outcomes.

Two studies have analyzed prospectively collected data for other purposes. ${ }^{45,63}$ Despite the use of robust outcome measures, the cross-sectional analysis and the convenience sample reduced confidence in the results of these studies. Other prospective studies recruit nonhomogeneous patient groups and assessing acute toxicity alone or evaluate patients in the early posttreatment period. ${ }^{64,65}$

Recently, Christianen et al ${ }^{66}$ developed predictive models for different swallowing problems observed in patients after curative (chemo)radiation, considering the most important dose-volume parameters and other pretreatment factors that determine physician-rated and patient-rated swallowing impairment. The study was prospectively designed and included 354 patients with head and neck cancer treated with (chemo)radiotherapy. The study found different predictive models for specific swallowing problems; for instance, problems with swallowing liquids were predicted best by dose to the supraglottic larynx, whereas problems with solid food were predicted best by the dose to the superior pharyngeal constrictor. Based on the results, the authors hypothesize that for greater food consistencies, the dose received by more cranial structures was predictive because greater pressure needs to be built to push the bolus down, and this head of pressure is generated more cranially. In routine clinical practice, however, the discussion on the importance of different dos$\mathrm{e}$-volume parameters and swallowing structures in determining dysphagia can be summarized as proposed by Eisbruch et $\mathrm{al}^{52}$ : the most critical structure depends on location of the target(s). For superiorly sited tumors (eg, oropharyngeal cancer) the most significant predictor of swallowing disorders is the dose to the superior constrictor muscle because this structure receives the highest doses, whereas in patients with laryngeal cancer, the larynx and inferior pharyngeal constrictors are at highest risk.

It is evident from the data presented in the table that 2 recent studies ${ }^{52,57}$ have avoided some of the methodologic shortcomings and identify IMRT as a valid strategy to reduce long-term dysphagia. If some of the ongoing trials on deescalating therapy for human papillomavirus-positive disease show good oncologic efficacy with less intensive treatment, the morbidity of posttreatment dysphagia could be reduced even further.

\section{Commentary on outcome measures}

This review has highlighted the use of outcome measures to assess swallowing function that we feel are not fit for that purpose. Measures such as gastrostomy retention rate and pharyngoesophageal stricture, especially when looked at on their own, are not robust. Several competing factors can be responsible for patients retaining their Gtubes. The interpretation of the presence of a stricture is variable on a contrast swallow or on rigid esophageal dilatation. Furthermore, a mild stricture may not necessarily be symptomatic for all patients. In the presence of several robust patient reported outcome measures, we believe that these should form an integral part of the outcomes assessment.

\section{CONCLUSIONS}

Although 5 of 6 studies have demonstrated that prophylactic swallowing exercises show benefit, further longitudinal studies are required to evaluate the clinical and cost benefit of prophylactic swallowing exercises and influencing factors. This will enable services to ensure optimal timing for swallowing intervention and direction of valuable resources.

It is likely that swallowing problems following radiotherapy increase with the addition of concomitant chemotherapy and with increased radiation dose to various structures that are part of the swallowing mechanism. The majority of studies published so far indicate that reducing the radiation dose to the larynx, pharyngeal constrictors, 
and cervical esophagus using IMRT appears to reduce the risk of swallowing dysfunction while minimizing the risk of a marginal miss so long as these structures are not part of the planned target volume. Additionally, the use of a G-tube may increase the probability of swallowing dysfunction. If adequate facilities are available for NG tube care and support during CRT, these should be avoided whenever possible. Rather than a one-size-fits-all approach to tube placement, a multidisciplinary evaluation based on each individual case is essential. This ensures that those who need early tube feeding are identified and receive appropriate support to optimize swallowing outcomes. Those not being tube fed at the outset should receive expert dietetic and swallowing intervention throughout treatment to ensure that their nutritional and hydration needs are met safely. Supportive care provision during treatment varies between centers ${ }^{7}$ and improved swallowing outcomes rely on multidisciplinary intervention, including psychological care and optimal analgesia. $^{67}$

Undoubtedly, all of the 3 strategies discussed here are resource intensive. Given the findings of this review, we believe that there is a clear trend for better swallow outcomes to be experienced. Clearly, more prospective studies from several settings, taking into account the drawbacks of the studies published so far, need to be performed analyzing each of the 3 strategies discussed here to generate more confidence in the results reported earlier.

\section{Acknowledgment}

Mr Justin Roe is funded by a grant from the Oracle Cancer Trust.

\section{REFERENCES}

1. Lewin JS. Dysphagia after chemoradiation: prevention and treatment. Int J Radiat Oncol Biol Phys 2007;69:S86-S87.

2. Wilson JA, Carding PN, Patterson JM. Dysphagia after nonsurgical head and neck cancer treatment: patients' perspectives. Otolaryngol Head Neck Surg 2011;145:767-771.

3. Nguyen NP, Frank C, Moltz CC, et al. Impact of dysphagia on quality of life after treatment of head-and-neck cancer. Int J Radiat Oncol Biol Phys 2005;61:772-778.

4. Trotti A, Pajak TF, Gwede CK, et al. TAME: development of a new method for summarising adverse events of cancer treatment by the Radiation Therapy Oncology Group. Lancet Oncol 2007;8:613-624.

5. Lazarus CL. Effects of chemoradiotherapy on voice and swallowing. Curr Opin Otolaryngol Head Neck Surg 2009;17:172-178.

6. Langmore S, Krisciunas GP, Miloro KV, Evans SR, Cheng DM. Does PEG use cause dysphagia in head and neck cancer patients? Dysphagia 2012;27:251-259.

7. Roe JW, Carding PN, Rhys-Evans PH, Newbold KL, Harrington KJ, Nutting CM. Assessment and management of dysphagia in patients with head and neck cancer who receive radiotherapy in the United Kingdom-a web-based survey. Oral Oncol 2012;48:343-348.

8. Krisciunas GP, Sokoloff W, Stepas K, Langmore SE. Survey of usual practice: dysphagia therapy in head and neck cancer patients. Dysphagia 2012;27:538-549.

9. National Institute for Clinical Excellence (NICE). Improving Outcomes in Head and Neck Cancer. London: NICE; 2004.

10. van der Molen L, van Rossum MA, Burkhead LM, Smeele LE, Rasch CR, Hilgers FJ. A randomized preventive rehabilitation trial in advanced head and neck cancer patients treated with chemoradiotherapy: feasibility, compliance, and short-term effects. Dysphagia 2011;26:155-170.

11. Carnaby-Mann G, Crary MA, Schmalfuss I, Amdur R. "Pharyngocise": randomized controlled trial of preventative exercises to maintain muscle structure and swallowing function during head-and-neck chemoradiotherapy. Int J Radiat Oncol Biol Phys 2012;83:210-219.

12. Kulbersh BD, Rosenthal EL, McGrew BM, et al. Pretreatment, preoperative swallowing exercises may improve dysphagia quality of life. Laryngoscope 2006;116:883-886.
13. Carroll WR, Locher JL, Canon CL, Bohannon IA, McColloch NL, Magnuson JS. Pretreatment swallowing exercises improve swallow function after chemoradiation. Laryngoscope 2008;118:39-43.

14. Chen AY, Frankowski R, Bishop-Leone J, et al. The development and validation of a dysphagia-specific quality-of-life questionnaire for patients with head and neck cancer: the M. D. Anderson dysphagia inventory. Arch Otolaryngol Head Neck Surg 2001;127:870-876.

15. Pauloski BR, Rademaker AW, Logemann JA, et al. Pretreatment swallowing function in patients with head and neck cancer. Head Neck 2000;22: $474-482$.

16. Stenson KM, MacCracken E, List M, et al. Swallowing function in patients with head and neck cancer prior to treatment. Arch Otolaryngol Head Neck Surg 2000;126:371-377.

17. van der Molen L, van Rossum MA, Ackerstaff AH, Smeele LE, Rasch CR, Hilgers FJ. Pretreatment organ function in patients with advanced head and neck cancer: clinical outcome measures and patients' views. BMC Ear Nose Throat Disord 2009;9:10.

18. Ahlberg A, Engstrom T, Nikolaidis P, et al. Early self-care rehabilitation of head and neck cancer patients. Acta Otolaryngol 2011;131:552-561.

19. Bjordal K, Hammerlid E, Ahlner-Elmqvist M, et al. Quality of life in head and neck cancer patients: validation of the European Organization for Research and Treatment of Cancer Quality of Life Questionnaire-H\&N35. J Clin Oncol 1999;17:1008-1019.

20. Zigmond AS, Snaith RP. The hospital anxiety and depression scale. Acta Psychiatr Scand 1983;67:361-370.

21. van der Molen L, van Rossum M, Smeele L, Rasch C, Hilgers F. Preventative rehabilitation in advanced chemo-radiated head and neck cancer patients; 2-year results. In: Proceedings of the 20th Annual Meeting of the Dysphagia Research Society; March 8-10, 2012; Toronto, Ontario, Canada; 2012.

22. Ricchetti F, Wu B, McNutt T, et al. Volumetric change of selected organs at risk during IMRT for oropharyngeal cancer. Int J Radiat Oncol Biol Phys 2011;80:161-168.

23. Kotz T, Federman AD, Kao J, et al. Prophylactic swallowing exercises in patients with head and neck cancer undergoing chemoradiation: a randomized trial. Arch Otolaryngol Head Neck Surg 2012;138:376-382.

24. Crary MA, Mann GD, Groher ME. Initial psychometric assessment of a functional oral intake scale for dysphagia in stroke patients. Arch Phys Med Rehabil 2005;86:1516-1520.

25. List MA, Ritter-Sterr C, Lansky SB. A performance status scale for head and neck cancer patients. Cancer 1990;66:564-569.

26. Focht K, Simpson K, Day T, Martin-Harris B. Markov modelling to evaluate pre-treatment swallowing exercises in head and neck cancer. In: Proceedings of the 20th Annual Meeting of the Dysphagia Research Society; March 8-10, 2012; Toronto, Ontario, Canada; 2012.

27. Retel VP, van der Molen L, Hilgers FJ, et al. A cost-effectiveness analysis of a preventive exercise program for patients with advanced head and neck cancer treated with concomitant chemo-radiotherapy. BMC Cancer 2011;11:475 (abstract).

28. Ackerstaff AH, Balm AJ, Rasch CR, et al. First-year quality of life assessment of an intra-arterial (RADPLAT) versus intravenous chemoradiation phase III trial. Head Neck 2009;31:77-84.

29. Rasch CR, Hauptmann M, Schornagel J, et al. Intra-arterial versus intravenous chemoradiation for advanced head and neck cancer: results of a randomized phase 3 trial. Cancer 2010;116:2159-2165.

30. Roe JW, Ashforth KM. Prophylactic swallowing exercises for patients receiving radiotherapy for head and neck cancer. Curr Opin Otolaryngol Head Neck Surg 2011;19:144-149.

31. Carnaby G, Lagorio L, Crary M, Amdur R, Schmalfuss I. Dysphagia prevention exercises in head and neck cancer: pharyngocise dose response study. In: Proceedings of the 20th Annual Meeting of the Dysphagia Research Society, March 8-10, 2012; Toronto, Ontario, Canada; 2012.

32. Lagorio L, Carnaby G. Impact of baseline factors on adherence to a preventative swallowing exercise (Pharyngocise) during CRT in head and neck cancer patients. In: Proceedings of the 20th Annual Meeting of the Dysphagia Research Society, March 8-10, 2012; Toronto, Ontario, Canada; 2012.

33. Langmore SE, Krisciunas GP. Dysphagia after radiotherapy for head and neck cancer: etiology, clinical presentation and efficacy of current treatments. Perspectives on swallowing and swallowing disorders (dysphagia). American Speech-Language-Hearing Association Division 2010;19: 32-38.

34. Paleri V, Patterson J. Use of gastrostomy in head and neck cancer: a systematic review to identify areas for future research. Clin Otolaryngol Allied Sci 2010;35:177-189.

35. Corry J, Poon W, McPhee N, et al. Randomized study of percutaneous endoscopic gastrostomy versus nasogastric tubes for enteral feeding in head and neck cancer patients treated with (chemo)radiation. J Med Imaging Radiat Oncol 2008;52:503-510.

36. Corry J, Poon W, McPhee N, et al. Prospective study of percutaneous endoscopic gastrostomy tubes versus nasogastric tubes for enteral feeding in patients with head and neck cancer undergoing (chemo)radiation. Head Neck 2009;31:867-876. 
37. Magne N, Marcy PY, Foa C, et al. Comparison between nasogastric tube feeding and percutaneous fluoroscopic gastrostomy in advanced head and neck cancer patients. Eur Arch Otorhinolaryngol 2001;258:89-92.

38. Mekhail TM, Adelstein DJ, Rybicki LA, Larto MA, Saxton JP, Lavertu P. Enteral nutrition during the treatment of head and neck carcinoma: is a percutaneous endoscopic gastrostomy tube preferable to a nasogastric tube? Cancer 2001;91:1785-1790.

39. Oozeer NB, Corsar K, Glore RJ, Penney S, Patterson J, Paleri V. The impact of enteral feeding route on patient-reported long term swallowing outcome after chemoradiation for head and neck cancer. Oral Oncol 2011; 47:980-983.

40. Chen AM, Li BQ, Lau DH, et al. Evaluating the role of prophylactic gastrostomy tube placement prior to definitive chemoradiotherapy for head and neck cancer. Int J Radiat Oncol Biol Phys 2010;78:1026-1032.

41. Kerstein R, Patterson J, Owen S, Johnson K, Cocks H. Severe hypopharyngeal stenosis following treatment for head a neck cancer-a case series In: 13th British Academic Conference in Otolaryngology 2009; Liverpool, UK; 2009.

42. Hutcheson KA, Lewin JS. Functional outcomes after chemoradiotherapy of laryngeal and pharyngeal cancers. Curr Oncol Rep 2012;14:158-165.

43. Nutting CM, Morden JP, Harrington KJ, et al. Parotid-sparing intensity modulated versus conventional radiotherapy in head and neck cancer (PARSPORT): a phase 3 multicentre randomised controlled trial. Lancet Oncol 2011;12:127-136.

44. Eisbruch A, Schwartz M, Rasch C, et al. Dysphagia and aspiration after chemoradiotherapy for head-and-neck cancer: which anatomic structures are affected and can they be spared by IMRT? Int J Radiat Oncol Biol Phys 2004:60:1425-1439.

45. Dornfeld K, Simmons JR, Karnell L, et al. Radiation doses to structures within and adjacent to the larynx are correlated with long-term diet- and speech-related quality of life. Int J Radiat Oncol Biol Phys 2007;68: 750-757.

46. Feng FY, Kim HM, Lyden TH, et al. Intensity-modulated radiotherapy of head and neck cancer aiming to reduce dysphagia: early dose-effect relationships for the swallowing structures. Int J Radiat Oncol Biol Phys 2007; 68:1289-1298.

47. Caglar HB, Tishler RB, Othus M, et al. Dose to larynx predicts for swallowing complications after intensity-modulated radiotherapy. Int $J$ Radiat Oncol Biol Phys 2008;72:1110-1118.

48. Mendenhall WM, Amdur RJ, Morris CG, Kirwan JM, Li JG. Intensitymodulated radiotherapy for oropharyngeal squamous cell carcinoma. Laryngoscope 2010;120:2218-2222.

49. Levendag PC, Teguh DN, Voet $\mathrm{P}$, et al. Dysphagia disorders in patients with cancer of the oropharynx are significantly affected by the radiation therapy dose to the superior and middle constrictor muscle: a dose-effect relationship. Radiother Oncol 2007;85:64-73.

50. Caudell JJ, Schaner PE, Desmond RA, Meredith RF, Spencer SA, Bonner JA. Dosimetric factors associated with long-term dysphagia after definitive radiotherapy for squamous cell carcinoma of the head and neck. Int $J$ Radiat Oncol Biol Phys 2010;76:403-409.

51. van der Laan HP, Christianen ME, Bijl HP, Schilstra C, Langendijk JA. The potential benefit of swallowing sparing intensity modulated radiotherapy to reduce swallowing dysfunction: an in silico planning comparative study. Radiother Oncol 2012;103:76-81.

52. Eisbruch A, Kim HM, Feng FY, et al. Chemo-IMRT of oropharyngeal cancer aiming to reduce dysphagia: swallowing organs late complication probabilities and dosimetric correlates. Int J Radiat Oncol Biol Phys 2011; 81:e93-e99.

53. Frowen J, Cotton S, Corry J, Perry A. Impact of demographics, tumor characteristics, and treatment factors on swallowing after (chemo)radiotherapy for head and neck cancer. Head Neck 2010;32:513-528.

54. Dabaja B, Salehpour MR, Rosen I, et al. Intensity-modulated radiation therapy (IMRT) of cancers of the head and neck: comparison of split-field and whole-field techniques. Int J Radiat Oncol Biol Phys 2005;63: 1000-1005.

55. Webster GJ, Rowbottom CG, Ho KF, Slevin NJ, Mackay RI. Evaluation of larynx-sparing techniques with IMRT when treating the head and neck. Int J Radiat Oncol Biol Phys 2008;72:617-622.

56. Caudell JJ, Burnett OL III, Schaner PE, Bonner JA, Duan J. Comparison of methods to reduce dose to swallowing-related structures in head and neck cancer. Int J Radiat Oncol Biol Phys 2010;77:462-467.

57. Schwartz DL, Hutcheson K, Barringer D, et al. Candidate dosimetric predictors of long-term swallowing dysfunction after oropharyngeal intensitymodulated radiotherapy. Int J Radiat Oncol Biol Phys 2010;78: 1356-1365.

58. Christianen ME, Langendijk JA, Westerlaan HE, van de Water TA, Bijl HP. Delineation of organs at risk involved in swallowing for radiotherapy treatment planning. Radiother Oncol 2011;101:394-402.

59. Mendenhall WM, Amdur RJ, Palta JR. Intensity-modulated radiotherapy in the standard management of head and neck cancer: promises and pitfalls. J Clin Oncol 2006;24:2618-2623.

60. Mendenhall WM, Mancuso AA. Radiotherapy for head and neck canceris the "next level" down? Int J Radiat Oncol Biol Phys 2009;73:645-646.

61. Wang JJ, Goldsmith TA, Holman AS, Cianchetti M, Chan AW. Pharyngoesophageal stricture after treatment for head and neck cancer. Head Neck 2012;34:967-973.

62. Feng FY, Kim HM, Lyden TH, et al. Intensity-modulated chemoradiotherapy aiming to reduce dysphagia in patients with oropharyngeal cancer: clinical and functional results. J Clin Oncol 2010;28:2732-2738.

63. Bhide SA, Gulliford S, Kazi R, et al. Correlation between dose to the pharyngeal constrictors and patient quality of life and late dysphagia following chemo-IMRT for head and neck cancer. Radiother Oncol 2009;93: 539-544.

64. Anand AK, Chaudhoory AR, Shukla A, et al. Favourable impact of intensity-modulated radiation therapy on chronic dysphagia in patients with head and neck cancer. Br J Radiol 2008;81:865-871.

65. Salama JK, Stenson KM, List MA, et al. Characteristics associated with swallowing changes after concurrent chemotherapy and radiotherapy in patients with head and neck cancer. Arch Otolaryngol Head Neck Surg 2008; 134:1060-1065.

66. Christianen ME, Schilstra C, Beetz I, et al. Predictive modelling for swallowing dysfunction after primary (chemo)radiation: results of a prospective observational study. Radiother Oncol 2012;105:107-114.

67. Rosenthal DI, Lewin JS, Eisbruch A. Prevention and treatment of dysphagia and aspiration after chemoradiation for head and neck cancer. J Clin Oncol 2006; 24:2636-2643.

68. Chen AM, Li BQ, Jennelle RL, et al. Late esophageal toxicity after radiation therapy for head and neck cancer. Head Neck 2010;32:178-183.

69. Fua TF, Corry J, Milner AD, Cramb J, Walsham SF, Peters LJ. Intensitymodulated radiotherapy for nasopharyngeal carcinoma: clinical correlation of dose to the pharyngo-esophageal axis and dysphagia. Int J Radiat Oncol Biol Phys 2007;67:976-981.

70. Lawson JD, Otto K, Grist W, Johnstone PA. Frequency of esophageal stenosis after simultaneous modulated accelerated radiation therapy and chemotherapy for head and neck cancer. Am J Otolaryngol 2008;29: 13-19.

71. Lee NY, O'Meara W, Chan K, et al. Concurrent chemotherapy and intensity-modulated radiotherapy for locoregionally advanced laryngeal and hypopharyngeal cancers. Int J Radiat Oncol Biol Phys 2007;69:459-468.

72. Li B, Li D, Lau DH, et al. Clinical-dosimetric analysis of measures of dysphagia including gastrostomy-tube dependence among head and neck cancer patients treated definitively by intensity-modulated radiotherapy with concurrent chemotherapy. Radiat Oncol 2009;4:52.

73. Peponi E, Glanzmann C, Willi B, Huber G, Studer G. Dysphagia in head and neck cancer patients following intensity modulated radiotherapy (IMRT). Radiat Oncol 2011;6:1. 